\title{
Multicore fibers: a novel platform for a robust and reconfigurable self- organization of light
}

\author{
Saurabh Jain ${ }^{1}$, Kunhao Ji ${ }^{1}$, Jayantha Sahu ${ }^{1}$, David.J.Richardson ${ }^{1}$, Julien Fatome ${ }^{2}$, Stefan Wabnitz ${ }^{3}$, \\ Massimiliano Guasoni ${ }^{1}$ \\ 1. Optoelectronics Research Centre, University of Southampton, Southampton SO17 1BJ, United Kingdom \\ 2. Laboratoire Interdisciplinaire Carnot de Bourgogne, CNRS, University of Bourgogne-Franche-Comte, 21078 Dijon, France \\ 3. Department of Information Engineering, Electronics and Telecommunications (DIET), Sapienza University, 00184 Rome, Italy
}

We report on novel dynamics of light self-organization based on the interaction amongst forward and backward supermodes of a multicore (MC) fiber (Fig.1). As compared to recent studies in standard single-core fibers [1,2], the MC platform offers many degrees of freedom - e.g. disposition of cores, core-to-core distance, relative size and shape of the cores etc- that pave the way to a rich mix of unexplored types of self-organization.

We demonstrate that a strong nonlinear regime can be achieved where the propagation of light is described by the following coupled Schrödinger equations: $\partial_{z} \mathrm{~F}_{n}+\mathrm{v}_{\mathrm{n}}{ }^{-1} \partial_{\mathrm{t}} \mathrm{F}_{\mathrm{n}}=-\mathrm{i} \gamma_{\mathrm{nn}}\left|\mathrm{F}_{\mathrm{n}}\right|^{2} \mathrm{~F}_{\mathrm{n}}+\mathrm{i} \mathrm{F}_{\mathrm{n}} \sum 2 \gamma_{\mathrm{nm}}\left(\left|\mathrm{Bm}_{\mathrm{m}}\right|^{2}+\left|\mathrm{F}_{\mathrm{m}}\right|^{2}\right)+\mathrm{i} \mathrm{B}_{n}{ }^{*} \sum \mathrm{m} \neq \mathrm{n}$ $2 \gamma_{\mathrm{nm}} \mathrm{B}_{\mathrm{m}} \mathrm{F}_{\mathrm{m}}, \mathrm{F}_{\mathrm{n}}(\mathrm{z}, \mathrm{t})$ and $\mathrm{B}_{\mathrm{n}}(\mathrm{z}, \mathrm{t})$ being the amplitude of the forward and backward supermode $n$ of the fiber, $\mathrm{v}_{\mathrm{n}}$ the related group velocity, $\gamma_{\mathrm{nm}}$ the Kerr intermodal nonlinear coefficient for supermodes $n$ and $m$ (a similar equation holds true for $\mathrm{B}_{\mathrm{n}}$ ). An example of the new self-organization dynamics is illustrated in Fig.2. Four independent sources are injected into the 4 cores of a MC fiber. A combination of 4 forward supermodes with time-varying random phase and power is excited. A CW-backward control beam CB, coupled to one supermode, is injected at the other end of the fiber. When the power of $\mathrm{CB}$ is large enough, an effective self-organization takes place that leads to the condensation of the forward field towards the supermode excited by the CB. A similar outcome is found when the $\mathrm{CB}$ is coupled to a combination of supermodes (reconfigurable self-organization). Most importantly, this process takes place independently of the number of cores and, due to the counter-propagating setup, it turns out to be robust against random geometrical imperfections of the fiber [3]. These results pave the way to novel devices for spatial division multiplexing and to high-power/highly scalable MC fiber lasers where independent optical sources, connected to different cores of the fiber, are all-optically and coherently combined.
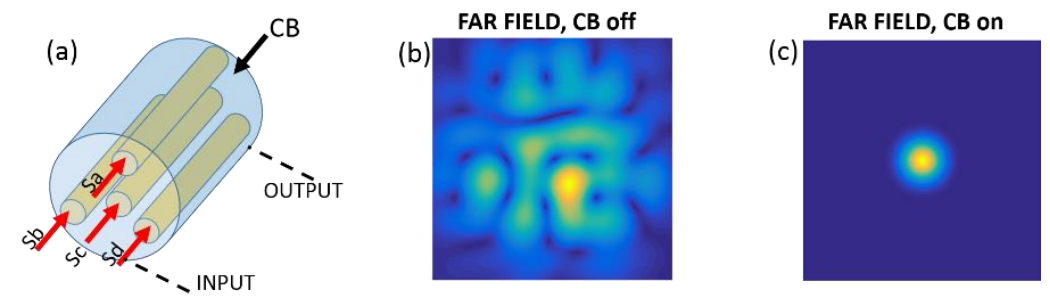

Fig. 1 A 4-core fiber is illustrated in panel a, with independent sources $\mathrm{Sa}, \mathrm{Sb}, \mathrm{Sc}$ and $\mathrm{Sd}$ feeding the cores at the input end. A backward control beam CB is injected at the output end and excites a supermode. If the CB is off, no self-organization takes place. The output forward supermodes preserve the original random relative phase and amplitude, which results in a random speckled far-field (panel b). If the CB is on and with sufficient power, a strong self-organization dynamics takes place. The forward field is attracted towards the supermode excited by the CB, which finally results in a high-quality and high-power density far-field (panel c). Similar results are obtained if CB excites a linear combination of supermodes, to which correspond different far-field configurations.
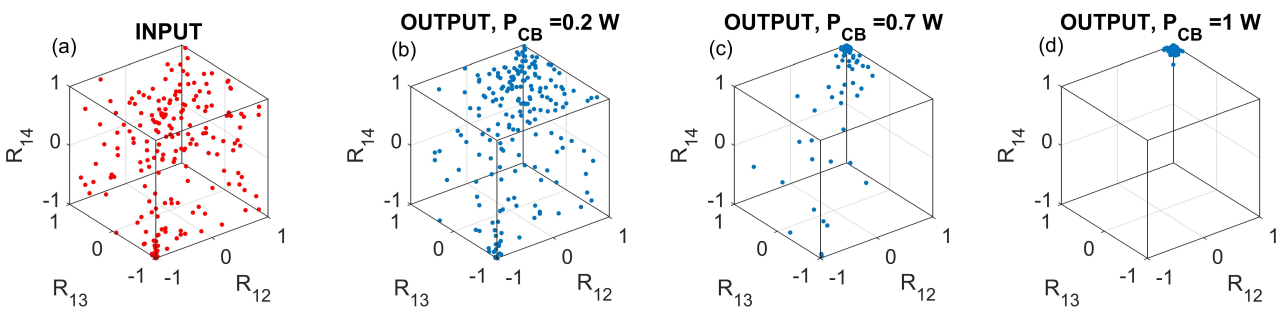

Fig. 2 Simulation of a 4-core, $5 \mathrm{~km}$ long fiber similar to the one represented in Fig.1a, with core diameter=5 um and core-to-central core distance $=10 \mathrm{um}$. Four independent sources with $250 \mathrm{~mW}$ average power excite a linear combination of forward supermodes at the input. A backward beam CB coupled to supermode 1 in injected at the output end (see fig.1). Rnm indicates the relative power between the forward supermodes $\mathrm{n}$ and $\mathrm{m}$. The input distribution of R12,R13 and R14 is random in time (200 temporal samples are represented in panel a). However, when increasing the power $\mathrm{Pcb}$ of $\mathrm{CB}$, the forward field undergoes an increasing attraction towards supermode 1 at the fiber output (see panels b,c,d where all samples collapse into R12=R13=R14=1).

\section{References}

[1] A. Picozzi, G. Millot, and S. Wabnitz, "Nonlinear virtues of multimode fibre," Nat. Photonics 9, 289-291 (2015).

[2] K. Krupa et al. "Spatial beam self-cleaning in multimode fibres", Nature Photon 11, 237-241 (2017).

[3] J.Fatome, S. Pitois, P.Morin, E.Assémat, E., D.Sugny, A. Picozzi, H.R. Jauslin, G.Millot, V.V.Kozlov, S.Wabnitz, “A universal optical all-fiber omnipolarizer", Sc. Reports 2, 1-8 (2012).

Funding: Horizon 2020 ERC Grant no. 740355, Horizon 2020 ERC Grant No. 802682, EPSRC EP/T019441/1 